\title{
Politique
}

\section{Les transformations idéologiques aux États-Unis entrevue avec Seymour Martin Lipset}

\section{Michèle Lamont}

Numéro 4, automne 1983

Crise et changements idéologiques

URI : https://id.erudit.org/iderudit/041000ar

DOI : https://doi.org/10.7202/041000ar

Aller au sommaire du numéro

Éditeur(s)

Société québécoise de science politique

ISSN

0711-608X (imprimé)

1918-6584 (numérique)

Découvrir la revue

Citer cet article

Lamont, M. (1983). Les transformations idéologiques aux États-Unis entrevue avec Seymour Martin Lipset. Politique, (4), 5-26.

https://doi.org/10.7202/041000ar d'utilisation que vous pouvez consulter en ligne.

https://apropos.erudit.org/fr/usagers/politique-dutilisation/ 


\title{
Les transformations idéologiques aux États-Unis entrevue avec Seymour Martin Lipset
}

\author{
Michèle Lamont \\ Stanford University
}

Seymour Martin Lipset est un des politologues américains qui a le plus marqué le développement de sa discipline au cours des dernières décennies. Son livre Political Man, the Social Bases of Politics $(1960 ; 1981)$ fut un événement déterminant pour le développement de l'analyse comparative en science politique et en sociologie. Il s'agissait d'une tentative originale d'incorporer des éléments structuraux à l'explication des relations politiques, particulièrement au niveau de l'analyse du comportement politique. Quand on sait l'importance qu'ont l'analyse comparative et la sociologie du développement dans l'univers intellectuel américain, on peut deviner l'impact de cette étude.

Lipset a participé très activement à l'édification de la culture sociologique contemporaine. Ses études sur l'université, sur le radicalisme aux États-Unis, sur les comportements politiques sont maintenant des classiques.

Après avoir enseigné entre autres à l'université de Toronto, à Harvard et à Berkeley, il détient maintenant la chaire Caroline S.G. Munro de science politique et de sociologie (Stanford University) et est Senior Fellow au Hoover Institution. 
Lipset discute ici des transformations du spectre politique américain, de la nouvelle droite, et du rapport entre classes et idéologies aux États-Unis. L'entrevue a été réalisée en mai 1983, à Palo Alto, Californie.

M.L. : Le thème général de cette entrevue est "changement politique et crise idéologique». Le concept d'idéologie est doté de plusieurs sens, tant dans le contexte américain que dans le contexte européen. Pouvez-vous préciser le sens que vous allez lui donner au cours de notre discussion?

S.M.L.: Comme vous le savez, le sens de ce concept s'est transformé avec le temps. Je crois que Napoléon fut le premier à l'utiliser dans l'histoire des idéologies. Puis Marx et les marxistes s'y sont référés. Chez Marx, ce concept renvoit aux croyances, aux systèmes d'idées du prolétariat ou des strates révolutionnaires, lesquelles défendent l'intérêt commun plutôt que les intérêts propres à un groupe. L'hypothèse du déclin des idéologies, pour sa part, apparait pour la première fois dans les écrits de Engels, quand il discute du dépérissement des idéologies sous le socialisme, à cause de l'absence de conflits de classes.

Le théoricien qui a le plus influencé l'analyse des idéologies est sans doute Karl Mannheim. Il s'est inspiré de la définition marxiste dans la mesure où par ce terme, il désigne un système de croyances propre aux groupes réactionnaires, particulièrement un système cherchant à maintenir les anciens états sociaux. Il oppose à ce terme l'utopie qui désigne les systèmes de croyances des groupes progressistes.

De manière générale, en sciences sociales, et au cours de discussions intellectuelles, l'usage qui est fait du concept d'idéologie ne renvoit pas à un système de croyances déterminé, que ce soit de droite, de gauche ou de centre. On désigne plutôt simplement un système intégré de croyances relatives surtout aux questions politiques. C'est ainsi que ce terme est généralement utilisé ici. 
On peut différencier les idéologies selon qu'elles sont plus ou moins cohérentes, organisées. Philip Converse qui est le meilleur analyste de sondages aux États-Unis a développé dans un article, il y a plusieurs années, une définition opérationnelle des idéologies basée sur des résultats de sondage. Entre autres, il analyse la cohérence idéologique des acteurs sociaux. Les interviewés sont considérés comme étant plus idéologiques s'ils sont plus cohérents, c'est-à-dire s'il est possible de prédire leurs réponses à une centaine de questions à partir de leurs réponses à deux ou trois questions.

La plupart des gens se rangent au centre du spectre; ils ne sont qu'à demi cohérents. Il est cependant intéressant de découvrir que plus les gens sont éduqués, plus ils sont cohérents, donc idéologiques. Inversement, les gens les moins éduqués sont moins constants dans leurs réponses, peut-être parce qu'ils sont moins informés ou intéressés. Selon ces résultats, nous nous dirigerions vers une société plus idéologique, la population étant de plus en plus éduquée, et ceci, tant à droite qu’à gauche.

M.L. : Comment conceptualiseriez-vous le rapport entre changement idéologique et crise économique?

S.M.L. : Si on examine ce qui se passe présentement au niveau mondial, il apparaît assez clairement que les événements économiques sont devenus le principal déterminant de la manière dont les gens réagissent. Ceci se vérifie particulièrement dans le cas du comportement électoral. Quand les conditions économiques sont défavorables, avec de forts taux de chômage et d'inflation, la population tend à incriminer le parti au pouvoir, qui tôt ou tard est forcé de démissionner. Ce phénomène s'est produit dans plusieurs pays où l'inflation et le chômage sont au-dessus de $10 \%$. Cela a provoqué l'abdication de gouvernements de droite et de gauche, socialistes ou conservateurs.

Prenons l'exemple de deux grands pays qui ont changé de direction politique au même moment, soit la France et les 
États-Unis. Ces deux pays avaient des indices de pauvreté similaires, un taux d'inflation similaire, assez élevé. Aux États-Unis, cette crise économique s'est produite sous Carter, et Reagan fut élu en 1980. Un de ses arguments électoraux était bien sûr qu'il allait améliorer la situation, chose que son adversaire n'avait pu faire au cours des années précédentes. En France, où il y avait un gouvernement conservateur, les conditions économiques pitoyables ont amené au pouvoir le premier gouvernement socialiste de l'histoire. Bien sûr aux États-Unis, certains prétendent, les conservateurs surtout, que la victoire de Reagan représente une victoire de la libre entreprise, une attaque contre le Welfare State. En France, on prétendait qu'il s'agissait d'une victoire du mouvement socialiste. Je pense plutôt que dans les deux cas, si on examine les résultats électoraux et les sondages, on découvre que les Américains n'ont pas voté pour la libre entreprise, ou les Français, pour le socialisme. Les Français ont voté contre Giscard, et les Américains, contre Carter. Par conséquent, il n'est pas question de conditions économiques poussant les électeurs vers la gauche ou la droite.

Un phénomène similaire s'est produit en Norvège et en Suède. En Norvège, les socialistes ont démissionné et les conservateurs sont venus au pouvoir. Quelques mois plus tard, en Suède, les conservateurs ont perdu le pouvoir et les socialistes sont rentrés. Dans les deux cas, les conditions économiques étaient pitoyables, et cela a amené un gouvernement socialiste en Suède, et un gouvernement conservateur en Norvège.

On peut évidemment analyser la question du rapport entre les conditions économiques et l'orientation idéologique d'un autre angle. Il y a plusieurs années, dans mon livre Political Man ${ }^{2}$, j'ai développé l'hypothèse selon laquelle les élections sont une

1. Lipset, S. M., Political Man, the Social Bases of Politics, Baltimore, John Hopkins University Press, 1981. 
forme de lutte de classes. C'était une hypothèse originale à l'époque. On découvre que dans presque tous les pays pour lesquels nous disposons de données, avec quelques exceptions, les partis de gauche, socialistes, communistes en Europe, démocrate aux États-Unis, sont soutenus par les secteurs les moins privilégiés de la population, les ouvriers et les moins éduqués. Les partis conservateurs, les Républicains ici, les Chrétiens-démocrates ailleurs, tirent leur support des fractions de la population les plus privilégiés.

Donc, dans chaque société, il y a un conflit qui s'exprime dans des élections démocratiques si elles sont tenues, ou hors des élections dans les sociétés autoritaires. Ce conflit met en scène la droite et la gauche qui sont bien sûr liées aux classes, aux positions dans la structure socio-économique. Et si on compare les programmes électoraux des Démocrates aux États-Unis, des Socialistes en France ou en Italie, des Partis Travaillistes en Grande-Bretagne ou en Australie, on découvre qu'ils partagent certaines caractéristiques. À des degrés divers, ils favorisent la distribution des privilèges dans la société, le partage des revenus, de l'influence sur le système social. Par opposition, les partis de droite défendent le statu quo économique.

Évidemment, l'intensité de ce conflit, la cohérence idéologique des partis varient de pays en pays, et historiquement à l'intérieur d'un même pays. Aux États-Unis et au Canada, les principaux partis politiques, de droite ou de gauche, tendent à être moins cohérents idéologiquement, même si ça a changé un peu au cours des dernières années. En Europe, les partis de gauche et de droite ont des idéologies plus cohérentes. Cependant, là aussi, avec la prospérité de l'après-guerre, il y a eu une tendance au déclin des idéologies, dans le sens de Converse. Les socialistes sont devenus moins socialistes, et les conservateurs, moins conservateurs. 
Un politologue, John Thomas, a fait il y a plusieurs années, une analyse de contenu des programmes électoraux des partis politiques du début du siècle jusqu'en 1960. Il a ensuite fait une mise à jour pour couvrir la période 1970. Son étude a mis en relief le fait qu'au cours de cette période de 1900 à 1970, les programmes politiques, des partis devenaient de plus en plus semblables, les différences s'estompant.

Les partis sociaux-démocrates tendent de plus en plus vers la droite. C'est d'ailleurs la critique qui leur est adressée par les radicaux. Ils ne se prétendent plus parti de classe, mais plutôt parti du peuple. La même chose se produit chez les socialistes. Thomas a démontré que les conservateurs se sont davantage transformés que les socialistes. Au début du siècle, les conservateurs étaient davantage contre le Welfare State, contre l'interventionnisme étatique, contre les services sociaux. Maintenant, jusqu'à tout récemment, ils acceptent davantage l'assurancemaladie, l'assurance-chômage, les logements subventionnés. Ils en sont venus progressivement à accepter les propositions keynésiennes relatives à l'intervention économique de l'État. Les socialistes ont aussi bien sûr modifié leur programme, en mettant moins l'emphase sur la nationalisation totale des moyens de production par exemple. Mais cependant, ils sont demeurés en faveur des syndicats, de la planification, des services sociaux. La gauche est donc allée moins à droite que la droite, à gauche.

Il faut souligner que depuis quelques années, il y a eu ce renouveau de la droite, qui rejoint la doctrine libérale classique. Je pense ici bien sûr à Thatcher, Reagan, Barre et Strauss en Allemagne. Ils sont tous retournés à la notion d'économie de marché, critiquant l'interventionnisme étatique, prétendant que le retour à la prospérité ne se fera qu'au prix de coupures. Aux États-Unis, sous Nixon et Eisenhower, qui étaient aussi Républicains, le Welfare-State s'est développé. Heath, le prédécesseur de Thatcher acceptait le Welfare-State. Il faut bien dire 
aussi que la gauche elle-même était devenue un peu plus critique du Welfare State, reconnaissant ses bienfaits, mais critiquant ses excès et ses effets sur l'économie.

M.L. : Diriez-vous que la polarisation idéologique est moins forte aux États-Unis maintenant?

S.M.L.: Je ne crois pas. Jusqu'en 1980, c'était probablement le cas. Sous Nixon, (ceci est souvent ignoré), plusieurs politiques dites de gauche ont été menées. Il a reconnu la Chine, a proposé la détente aux Soviets, etc. Les conservateurs prétendaient pouvoir faire ce que les socialistes voulaient faire. Au niveau de la politique intérieure américaine, c'est sous Nixon que le mouvement "Affirmative Action» a été mis en place. Cette politique impose des quotas d'engagement pour les minorités, les femmes, les noirs. Elle fut mise en place en 1969, par le Labor Department de Nixon, et plus tard, celui-ci proposa d'étendre cette politique à l'université etc... Et ce n'est pas là une politique du Congrès, mais une politique de l'administration Nixon. En outre, le Président développa des politiques de sécurité au travail, à la demande des syndicats. Des choses similaires se sont produites sous Eisenhower. Peut-être que ça s'explique comme tentative des conservateurs pour aller chercher l'électorat de l'opposition.

Reagan représente un phénomène différent, et il ne s'agit pas d'un phénomène nouveau. La nomination de Goldwater en 1964 représentait le même genre de chose. Si on cherche à découvrir ce que Reagan représente en termes de classes, on découvre qu'il est principalement lié aux petites entreprises, à la classe moyenne indépendante. Il a aussi beaucoup de support de la part des cols blancs, mais pas tellement de la part de la « Big Business». En 1980, lors du congrès républicain pour la nomination présidentielle, le candidat de la «Big Business», celui qui était le plus financé par ce groupe était John Connally. Il n’a pas eu un gros succès, même s'il disposait de plus d'argent que 
Reagan, de plus d'argent que les autres candidats supportés par d'autres groupes. Et en outre, il était le candidat le plus favorable à l'intervention étatique. Il était favorable au maintien du contrôle étatique, à la planification, au Welfare State. La « Big Business» en était venue à accepter l'intervention étatique comme leur étant favorable.

La nouvelle droite qui s'identifie ici à Reagan est anti «Big Business». Elle regroupe les petites et moyennes entreprises, les nouveaux riches, souvent des gens très fortunés. Mais il ne s'agit pas de grosses corporations, plutôt des corporations de taille moyenne.

Un phénomène similaire est survenu en Angleterre. La Federation of British Industries qui est l'organisation des grandes corporations anglaises n'était pas favorable au programme de Thatcher. La tendance favorisant les théories économiques de Friedman ne provient pas de la "Big Business»

Si on examine les sondages, on découvre que les politiques économiques de Reagan et de Thatcher ne sont pas très populaires. La plupart des Américains, y compris les Républicains, favorisent le Welfare State. Richard Wirthlin, qui faisait des sondages pour Reagan au cours de la campagne électorale, racontait que le Président était plus anti-étatique que la majorité des Américains, que la majorité des Républicains, et qu'il a dû faire des compromis afin d'être élu. La majorité des gens qui supportent les politiques reaganniennes affirment, lorsqu'on leur demande si l'État doit aider les défavorisés, qu'ils sont favorables à de telles politiques.

Il est intéressant de se demander comment il se fait que des points de vue qui ne sont pas très populaires, soient devenus si puissants, aux États-Unis et en France. Un économiste français qui est un ami de Barre, me racontait que ce dernier était assez favorable aux théories de Friedman, mais qu'il n'oserait 
jamais les appliquer, de peur de devoir démissionner le lendemain.

Ce qui s'est probablement passé dans le cas de Reagan c'est que l'économie était tellement en mauvaise posture, qu'il fallait un changement. On a fait de l'État le responsable de la crise, on a proposé une solution. Je ne pense pas qu'il s'agissait d'une conspiration de la «Big Business». Les conservateurs n'ont pas gagné parce qu'ils avaient plus d'argent, mais plutôt parce qu'ils étaient plus sûr d'eux, parce qu'ils étaient plus efficaces. Les intellectuels, particulièrement les économistes, ont eu un impact considérable. Les économistes se sont radicalement tournés vers la défense du marché. Les professeurs, mêmes Démocrates, et certains socialistes, défendent le marché, écrivent contre le Welfare State, analysent le problème de l'inflation en en rendant l'État responsable. Peut-être que l'explication la plus simple du reagannisme est une réaction à l'inflation.

M.L. : Dans votre nouveau livre The Confidence Gap ${ }^{2}$, vous démontrez qu'il y a aux États-Unis une crise de confiance à l'égard de toutes les institutions, qu'il s'agisse des syndicats, de la «Big Business», ou des gouvernements. Comment l'expliquez-vous?

S.M.L. : Entre 1945 et 1965, dans cette période de prospérité, les gens étaient de plus en plus optimistes, confiants à l'égard des institutions, particulièrement à l'égard des institutions politiques. À la même époque, le Welfare State se mettait en place, c'était l'abondance. À la fin des années ' 60 , il y a ce déclin de confiance qui est reflété par les sondages. Ils démontrent tous une immense baisse du taux de satisfaction de la population, et ceci à l'égard de toutes les institutions, politiques, militaires, étatiques, les corporations, les syndicats... Ce n'était pas là tant

2. Lipset, S. M., Schneider, W., The Confidence Gap, Business, Labor and Government in Public Mind, New York, The Free Press, 1983. 
le produit d'une crise économique que d'une crise d'autorité dûe à la guerre du Vietnam. Au même moment, vous avez le mouvement contre la guerre, les révoltes noires, le mouvement féministe, les révoltes des étudiants, le mouvement des homosexuels etc. La fin des années ' 60 était une période de bouillonnement et de chaos. La société se décomposait à travers une immense crise d'autorité, comme le montrent les sondages de l'époque. Ce n'était guère surprenant. Ce qui était surprenant plutôt, c'était l'intensité du mouvement, en terme de pourcentage de personnes qui changeaient leur attitude à l'égard des institutions.

Si on examine les sondages de 1971 à 1983, il y a une baisse graduelle de confiance, mais ce mouvement n'est pas très marqué. On a l'impression que les gens veulent espérer, veulent avoir une raison de croire. Mais chaque fois que le mouvement va à la hausse, un événement survient, le Watergate, la crise... Dans notre livre, Schneider et moi avons analysé la relation entre la crise de confiance et les indicateurs économiques. Il appert clairement que quand l'inflation et le chômage augmentent, la confiance baisse. Le chômage a cependant plus d'effet que l'inflation.

La légitimité des institutions elles-mêmes n'a pas diminué. Les Américains ont foi aux institutions, mais n'ont pas confiance à la manière dont elles sont administrées. Généralement, ils trouvent que leur situation personnelle ne s'est pas détériorée, sauf pour les chômeurs au cours des trois dernières années. Ce n'est pas leur position personnelle qui est en question, mais la façon dont les institutions sont administrées, la façon dont la société fonctionne. Cette crise ne produit pourtant pas de mouvement de contestation.

M.L. : Pourquoi pensez-vous qu'il en est ainsi?

S.M.L. : Peut-être parce que les gens ne se sentent pas affectés personnellement. Par conséquent, ils ne réagissent pas. Ils ne 
deviennent pas conservateurs... Ils deviennent plutôt cyniques, ce qui nous ramène à la question des idéologies.

Jusqu'à la deuxième guerre mondiale, les gens croyaient qu'il était possible de faire des changements fondamentaux, de produire une meilleure société, et ce, à travers le socialisme, le fascisme... La possibilité du différent existait, ainsi que la possibilité que les choses s'améliorent. Maintenant, le fascisme a complètement été délégitimé comme système social, avec la guerre et l'holocauste. Le socialisme a aussi perdu son aura. Il existe une grande variété de gouvernements sociaux-démocrates, socialistes, et on ne sait si les choses sont mieux ou pires dans ces pays. Il est clair que personne ne prétend que l'utopie socialiste a été réalisée. Plus personne ne considère ces systèmes comme pouvant produire des changements radicaux, même chez les militants socialistes ou communistes je crois... Il y a ce sentiment que nous avons tenté toutes sortes de choses, le fascisme, le socialisme, le Welfare State et aucune de ces idéologies n'a produit le bon système que nous espérions. Le cynisme politique s'installe, et bien sûr, la participation électorale chute. Les gens ne cherchent plus la bonne solution. Ils croient qu'elle n'existe pas.

M.L. : D'où le mouvement de dé-politisation?

S.M.L.: Oui... et de cynisme...

M.L. : Alors les gens se réfugient dans leur vie privée...

S.M.L.: Oui, et spécialement parce que leur vie privée n'est pas si mal en général. Le niveau de vie a augmenté. Bien sûr, il y a des pauvres, mais il ne s'agit pas d'une classe. Ce sont des personnes âgées, des mères célibataires... Les pauvres ne sont plus des pauvres ouvriers. Ce sont des groupes spécifiques inorganisés, qui ne peuvent avoir d'impact politique. Les autres ont leur voiture, leur télévision, leurs enfants vont à l'école plus longtemps... Ce n'est pas si mal. 
M.L. : Voyez-vous des différences dans la forme que prend la crise de confiance par rapport aux syndicats et à la "Big Business"?

S.M.L.: Les syndicats sont sans doute l'organisation qui est dans la posture la moins favorable. Les syndicalistes se situent au bas des échelles de prestige... Peut-être que les vendeurs de voitures usagées viennent un peu après eux... Les Américains ne tendent pas à avoir une opinion favorable des syndicats. Simultanément, ils croient que ce sont des institutions nécessaires, pour la protection des travailleurs. Mais ces syndicats sont vus comme étant trop corrompus, compte tenu de leurs rapports avec le crime organisé etc...

Je pense aussi que les syndicats sont considérés comme étant trop puissants. Ils constituent une seule entité, un seul mouvement. L'AFL-CIO est considérée comme une chose unifiée. Par opposition, au niveau de l'industrie, c'est divisé. Il y a General Motor, les banques, les compagnies de téléphone. Il n'y a pas la même unité. Les corporations s'affrontent et se limitent les unes les autres. Quand on questionne les gens, on découvre qu'ils sont contre la «Big Business», mais le «Big Labor» est une force plus visible; il est considéré comme étant trop puissant.

Ce phénomène est également lié à la nature des classes sociales aux États-Unis. Je travaille sur un livre intitulé $W$ hy no socialism in the United States? ${ }^{3}$ Je cherche à comprendre pourquoi les États-Unis est un des seuls grands pays industrialisés n'ayant pas un important mouvement socialiste organisé. Et la réponse se trouve partiellement dans la nature de la structure de classe des États-Unis... Il me faudrait beaucoup de temps pour développer là-dessus mais...

3. Lipset, S. M. "Why no Socialism in the United States?" in Sources of Contemporary Radicalism, Bialer S., Sluzar, S., eds., Westview Press, 1977. 
Si on examine la nature des relations de classes aux ÉtatsUnis, on découvre qu'au niveau des relations inter-personnelles les relations de classes sont beaucoup moins hiérarchiques. Les Américains ne savent pas vraiment comment procéder avec le personnel domestique par exemple. Ils traitent les serveurs de restaurant de façon amicale, alors qu'en Europe, la différence est clairement établie.

Ici, bien sûr, il n'y a pas d'égalité de revenu, ou d'égalité sociale, mais il y a en quelque sorte une volonté d'égalité de respect. On veut que chaque personne soit traitée comme un individu. C'est évident dans la manière dont on parle d'habitude aux gens des classes défavorisées par exemple.

Ceci est sans doute lié à la nature des relations capitalistes ici. En Europe, lors de la naissance de la classe ouvrière, il n'était nullement nécessaire de convaincre les ouvriers qu'ils étaient ouvriers. Dans une société post-féodale, où l'emphase est mise sur les classes sociales, sur les hiérarchies, tout incite à penser en termes de classe. Le système électoral lui-même y défnissait les ouvriers comme tels. Aux États-Unis, ce n'était pas le cas. Les socialistes devaient convaincre la classe ouvrière, lui enseigner qu'elle est une classe. Il est vrai qu'après la Grande Dépression de 1929, les États-Unis se sont davantage dirigés vers une conscience de classe dans. le sens européen du terme, jusqu'après la guerre. Et nous n'avons cependant toujours pas de mouvement socialiste. Il est intéressant de remarquer que la participation des ouvriers au mouvement syndical a atteint son sommet aux États-Unis au milieu des années ' 60 , alors que 33\% environ des travailleurs appartenaient à un syndicat. Maintenant, c'est tombé à $20 \%$

M.L. : Pourquoi la notion de groupe d'intérêt, de "constituency » est-elle si importante dans la sociologie politique américaine? 
S.M.L. : D'abord, il faut savoir que les «constituency» renvoient à deux choses: aux circonscriptions électorales et aux groupes de pression. Les groupes de pression ont un impact énorme à cause de la manière dont le système politique américain fonctionne. Au niveau des élections présidentielles, l'ensemble du pays forme une seule circonscription électorale. Il y a deux candidats principaux, autour desquels se forment des coalitions très hétérogènes. Ces coalitions se forment avant les élections. Les partis eux-mêmes sont très peu définis, contrairement à ce qui se passe en Europe. Par exemple, les membres du Congrès votent non pas avec leur parti, ou avec le gouvernement, mais en fonction des groupes d'intérêt dont ils reçoivent l'appui.

On peut évidemment identifier des caractéristiques générales pour les deux principaux partis, les Démocrates étant plus à gauche, et les Républicains plus à droite, mais ça demeure très hétérogène. Les partis ne vont pas nécessairement prendre des positions et essayer de réaliser leurs promesses. Ce qui se passe plutôt est la chose suivante: les groupes de pression qui veulent faire changer des choses forment un mouvement, généralement organisé autour d'un seul objectif. Puis, ils cherchent à influencer l'appareil politique, à travers le lobbying. C'est une vieille tradition dans la vie politique américaine. On n'a qu'à penser aux abolitionnistes, aux partisans de la prohibition, au mouvement pour la paix, au mouvement des suffragettes. On a donc deux partis et plusieurs mouvements, alors qu'en Europe, il y a plusieurs partis, et moins de mouvements.

Les groupes d'intérêt agissent de deux manières. La première et la plus conventionnelle, est le lobbying: les individus se regroupent et poussent sur le système politique, offrant leur vote à ceux qui vont soutenir les changements qu'ils proposent. Le contrôle des armes à feu est un bon exemple. The National Rifle Association est composé principalement de chasseurs pour 
qui les armes à feu sont une passion. Ils ne veulent supporter aucune limite. Ils disposent de listes informatisées de leurs supporters dans toutes les circonscriptions. Ils vont voir les membres du Congrès et les menacent de leur retirer tous ces votes s'ils se prononcent en faveur du contrôle des armes à feu. La majorité des Américains sont en faveur du contrôle dans une proportion de deux pour un; les membres du Congrès aussi. Cependant, ils ne voteront jamais en faveur du contrôle parce qu'ils craignent de perdre leurs votes. Ceci est un cas classique.

Ainsi, les groupes de pression organisés autour d'une seule question peuvent avoir un impact énorme sur les décisions politiques. Il en est ainsi partiellement parce que le reste de la population demeure inorganisé. La même chose se produit dans le cas des relations extérieures ou de la politique des armements. Les minorités peuvent avoir un immense impact. Ici, à San Francisco par exemple, les homosexuels représentent 15 à $20 \%$ de la population et sont considérés comme une minorité aussi importante que les noirs ou les chicanos. Ils sont organisés et les politiciens sont forcés de réagir, d'instaurer des politiques qui les favorisent de manière à gagner leurs votes.

L'autre méthode utilisée par ces groupes de pression fut bien sûr la désobéissance civile. Ce fut le cas avec les abolitionnistes avant la guerre civile, avec les prohibitionnistes qui allaient casser les saloons, avec le mouvement anti-guerre, le mouvement des suffragettes... Dans ces cas, les groupes se sentent tellement concernés par certaines questions qu'ils refusent de laisser la société fonctionner tant qu'ils n'auront pas obtenu satisfaction. Si le mouvement est assez important, et s'il peut causer assez de dégats, alors ces groupes obtiennent ce qu'ils cherchent. Lors du Civil Rights Movement, Lyndon Johnson a affirmé explicitement que le pays était en train de tomber en morceaux, et qu'il était impossible de ne pas voter... faute de quoi le pays n'aurait pas tenu le coup. 
Il est intéressant de remarquer que les gens qui participent à ces mouvements sont souvent les plus éduqués, les plus sophistiqués politiquement. Comme il y a eu une augmentation importante du niveau de scolarité de la population, on peut s'attendre à ce que ces mouvements organisés autour d'une seule question deviennent plus nombreux et plus puissants. Et ces groupes ne sont pas unis politiquement. Ce ne sont évidemment pas des classes. Leur orientation politique correspond généralement à ce que leurs partisans ont étudié à l'école. Ceux qui sont issus des sciences sociales tendent à être plus à gauche que les diplômés des facultés de commerce, d'agriculture et de médecine par exemple. Mais en général, ces groupes tendent à être plus sophistiqués, articulés, que le reste de la population, peu importe leur orientation politique.

On peut vraiment s'attendre à ce que ces groupes deviennent plus importants, au même moment où la participation électorale diminue. Je pense aussi que ces mouvements se sont étendus à l'Europe, en provenance des États-Unis.

M.L. : Êtes-vous certain qu'il s'agisse d'une diffusion à partir des États-Unis?

S.M.L.: Je pense que oui... Je ne dis pas que ces mouvements ne se seraient pas produits si les mouvements américains n'avaient pas existé, mais l'expérience américaine a certainement eu un impact important ailleurs. En bonne partie, ce que nous appelons maintenant la "New Politics» a émergé aux ÉtatsUnis. Ceci peut s'expliquer entre autres par la façon dont les médias fonctionnent ici. Par la télévision, les choses sortent plus facilement des États-Unis qu'elles n'y entrent.

M.L.: Le rôle joué par ces groupes de pression dans la vie politique américaine indique-t-il que les gens se sentent davantage légitimés à intervenir dans la vie politique? Qu'ils ont le droit de produire leur société, de définir ses enjeux, compte tenu de l'importance de la tradition démocratique ici? 
S.M.L. : Je pense que oui. Ça a aussi quelque chose à voir avec le populisme, qui est plus important ici qu'en Europe. Je lisais hier un article dans le New York Times à propos des différences entre le Canada anglais et les États-Unis. Comme vous le savez, j'ai passé quelques années à Toronto, et en Saskatchewan. À l'époque, j'ai aussi écrit un article comparant le Canada anglais et les États-Unis ${ }^{4}$. Je disais alors que le Canada était un pays d'ordre et d'autorité par comparaison.

M.L. : Et le Québec?

S.M.L.: Je connais moins, Mais je pense qu'avant la Révolution tranquille, c'était aussi une région plus stable que les États-Unis. Les États-Unis sont un pays de révolution, où la révolution a réussi. Le Canada est aussi né d'une révolution, mais c'est le pays de la contre-révolution, à cause de l'immigration des loyalistes. C'est le pays qui a maintenu la monarchie. On y conserve les symboles de la tradition, des valeurs hiérarchiques. Le Canada anglais a rejeté la révolution américaine.

Le Québec est aussi un pays de contre-révolution. Il n'était plus partie de la France lors de la révolution bourgeoise. Mais l'Église catholique, institution dominante, a rejeté cette révolution à cause de son anti-cléricalisme... Et comme les élites économiques et intellectuelles avaient fui le pays après la Conquète...

L'Église cherchait clairement à maintenir les idées françaises hors du Québec. S'il n'y avait pas eu la Révolution française, le Québec serait probablement resté plus près de la France, et aurait souhaité plus rapidement se débarrasser de la monarchie anglaise. Mais pour l'Église, cette monarchie représentait un moindre mal.

Donc, historiquement, le Québec a rejeté la Révolution française de la même manière que le Canada anglais a rejeté la

4. Lipset, S. M., Agrarian Socialism, University of California Press, 1950; Lipset, S. M., Revolution and Counterrevolution, New York, Basic Books, 1968. 
Révolution américaine. Chacun a tenté de maintenir ce qui existait auparavant, l'état pré-révolutionnaire au sens de Mannheim. Ces valeurs se sont institutionnalisées dans l'obéissance à l'État, dans le respect des hiérarchies etc...

M.L. : Vous savez que les références à l'Église ont été disqualifiées comme facteur explicatif au Québec.

S.M.L.: Oui, les choses ont changé depuis la Deuxième Guerre Mondiale. L'Église elle-même s'est transformée. On ne peut plus dire que c'est un pays contre-révolutionnaire. Les Canadiens anglais ont longtemps considéré que les Américains étaient populistes, grossiers, égalitaires. Ils avaient une attitude élitiste à leur égard. Ce n'est plus tellement le cas. Plusieurs Canadiens et Québécois se considèrent même à gauche des Américains. Mais simultanément, il me semble que le Canada anglais demeure plus «law and order». Par exemple, le taux de criminalité, le pourcentage de policiers et d'avocats per capita demeure plus faible au Canada qu'aux États-Unis. Le pourcentage de détenteurs d'assurance-vie est plus élevé aussi. Il y a toutes sortes d'indicateurs de ces différences au niveau de la structure sociale et des valeurs. Bien sûr, les choses se sont transformées plus rapidement au Québec qu'au Canada anglais. La tradition hiérarchique y est moins forte.

Jusqu'à tout récemment, de nombreux Nord-Américains avaient une image agraire du Québec. On voyait les Québécois presque comme des paysans féodaux. Ce n'est plus du tout vrai. On découvre qu'il s'agit d'une société nord-américaine, qu'on a maintenant des «Yankees» francophones, des Nord-Américains francophones, et non pas des Français vivant en Amérique. Les Québécois, il me semble, sont d'ailleurs plus proches des Américains. Je pense qu'il y a plus de différence entre le traditionnel Canadien anglais et l'Américain qu'entre l'Américain et le Québécois. Alors nous avons des "Yankees» francophones au Québec. 
M.L. : Et qu'est-ce que vous faites de notre spécificité culturelle?

S.M.L.: Je pense que la chose la plus importante est que les Québécois sont des Américains, et que le Canada doit refléter ce fait puisque ce pays n'est pas simplement composé de Canadiens anglais. Certainement, la langue française, la culture française sont très différentes.

Il y a une qualité similaire pour tous les pays d'Amérique qui les rendent différents d'une même manière par rapport à l'Europe. Les pays d'Amérique Latine, le Canada, les États-Unis sont différents d'une même façon. Ils font parti du Nouveau Monde pour ainsi dire.

Il y a quelque temps, je parlais avec Henrique Cardoso, le sociologue brésilien, à propos des facteurs qui font qu'il n'y a pas de socialisme aux États-Unis. Il m'affirma qu'un phénomène similaire existe au Brésil. En Amérique, on ne peut avoir le même type de politique de classe qu'en Europe, parce qu'il n'y a pas ce passé féodal. On ne peut construire de mouvement socialiste selon le modèle européen. Dans tous les cas, Amérique Latine, États-Unis, Québec, Canada, les mêmes choses manquent. Et en même temps, tant dans le cas du Québec que dans celui du Brésil, ils ont une culture spécifique nettement affirmée.

Une des choses qui me frappe de la culture québécoise est celle-ci: j'étais au Québec au milieu des années '60, et un ami m'annonça fièrement qu'il était maintenant possible de se procurer Le Monde tous les matins à Montréal, et L'Express, etc. Je me suis alors dis: si vous pensez que c'est là la chose la plus extraordinaire de la vie intellectuelle au Québec, le fait de pouvoir recevoir tout de Paris en quelques heures, vous êtes perdus. Vous êtes un satellite, vous êtes Bordeaux ou Lyon, vous êtes dans le désert français. Montréal est une capitale, une métropole 
intellectuelle indépendante, et non pas une ville française située à des milliers de milles de Paris.

Je pense que cette période est maintenant heureusement finie. Les Québécois ne prennent plus l'accent français. Le Québec est un pays indépendant culturellement, comme l'Australie. Il y a cependant plusieurs éléments américains. Le Québec a conservé de nombreux éléments du modèle français traditionnel. Par exemple, les Québécois sont moins avides d'argent, sont plus orientés vers la culture; certains hommes d'affaire écrivent des romans. Les Québécois sont plus intéressés à la vie culturelle.

M.L. : En guise de conclusion, pouvez-vous tracer un portrait général des rapports entre classes d'une part, et orientation politique et idéologique aux États-Unis d'autre part?

S.M.L. : Aux États-Unis, la plupart des conflits de classe sont des conflits opposant ceux qui sont au pouvoir et ceux qui ne le sont pas. Ces conflits opèrent de la même façon qu'ailleurs, même s'ils ne sont pas conceptualisés de la même manière. Les Démocrates et les Républicains sont différents, les Démocrates sont plus proches des syndicats etc...

Il y a des différences de classes, si vous considérez les attitudes. Il y a correspondance entre ce que les gens pensent et leur statut socio-économique, particulièrement au niveau des questions économiques - taxation des revenus, droit des travailleurs, assurance-santé etc. En ce qui concerne les questions sociales - relations familiales, avortement, la place de la religion dans les relations internationales - les positions de classe semblent être moins déterminantes, quoique les attitudes les plus libérales tendent à être défendues par les gens les plus éduqués, qui sont aussi généralement les plus fortunés.

Ici, les différences de classe comme explication des conflits idéologiques n'ont jamais été acceptées comme facteur légitime, sauf par une minorité. C'était moins le cas au cours des années 
'30 et '40. Par exemple, tant Roosevelt que Truman ont attaqué Wall Street et la «Big Business». Mais ils n'opposaient pas prolétariat et capitalistes, capital et travail. Ils s'opposaient aux excès des corporations.

Je pense que les expressions conscientes des oppositions de classe ont vraiment décliné depuis les 30 dernières années. Ceci se reflète clairement dans le mouvement syndical. Les gauchistes espèrent que la classe ouvrière va s'organiser, mais ça ne se produit pas. Je pense que tant ici qu'en Europe, le réformisme radical, ce que les gens appellent la politique de la «New Class» gagne de l'importance.

Les fractions éduquées de la population, l'intelligentsia va se battre contre le nucléaire, pour les droits civiques, pour le droit des femmes... Ils veulent une société plus propre, une société plus participationiste. Ils ne sont pas intéressés aux classes ou aux syndicats. Ceux-ci, tout comme la "Big Business" sont considérés comme étant trop égoïstes, intéressés uniquement à faire plus d'argent. La «New Class" (même si ce terme n'est pas approprié et désigne mal ce que l'on veut dire) est plutôt intéressée à la qualité de la vie, ou à la paix internationale. Ils se considèrent de gauche, ou démocrates, de la même façon que les Verts en Europe. Mais il ne s'agit aucunement d'un radicalisme de type marxiste. Il s'agit d'une nouvelle forme de radicalisme. Si on cherche à analyser ce phénomène à travers le schéma marxiste classique, ça ne fonctionne pas.

M.L. : C'est pourquoi dans Dialogues on American Politics ${ }^{5}$ vous mettez en rapport le statut socio-économique avec les attitudes à l'égard des questions sociales d'une part, et les questions économiques d'autre part?

S.M.L.: Oui, et cette distinction est spécialement importante dans les périodes de prospérité. Quand les choses vont mal, les

5. Horowitz, 1. L., Lipset, S. M., Dialogues on American Politics, Oxford, Oxford University Press, 1978. 
gens retournent évidemment aux problèmes économiques, et les enjeux sociaux perdent leur importance.

M.L. : Il y a cette histoire qui raconte qu'aux États-Unis, le mot «classe» est un tabou de la même façon que l'est le mot "sexe» en Angleterre...

S.M.L. : Peut-être qu'ici, les deux mots sont un tabou. On en est toutefois pas encore à faire un usage politique du mot sexe... 